\title{
Recombinant human hyaluronidase-facilitated subcutaneous immunoglobulin infusion in primary immunodeficiency diseases
}

\author{
Richard L Wasserman ${ }^{*}, 1$ \\ ${ }^{1}$ Allergy Partners of North Texas, Medical City Children's Hospital, Dallas, TX, USA \\ * Author for correspondence: drrichwasserman@gmail.com
}

\begin{abstract}
Most primary immunodeficiency diseases (PIDDs) resulting in antibody deficiency require intravenous or subcutaneous immunoglobulin $\mathrm{G}(\mathrm{SCIG})$ replacement therapy. The flow and distribution of SCIG to the vasculature is impeded by the glycosaminoglycan hyaluronan in the extracellular matrix, which limits the infusion rate and volume per site, necessitating frequent infusions and multiple infusion sites. Hyaluronidase depolymerizes hyaluronan and is a spreading factor for injectable biologics. Recombinant human hyaluronidase ( $\mathrm{rHuPH} 20$ ) increases SCIG absorption and dispersion. In patients with PIDD, SCIG facilitated with rHuPH20 (IGHy) has been shown to prevent infections, be well-tolerated and reduce infusion frequency and number of infusion sites as compared with conventional SCIG. This article reviews IGHy clinical studies and real-world practice data in patients with PIDD.
\end{abstract}

First draft submitted: 16 July 2017; Accepted for publication: 16 August 2017; Published online: 5 September 2017

Keywords: immunoglobulin $\bullet$ intravenous administration of immunoglobulin $\bullet$ primary immunodeficiency disease $\bullet$ recombinant human hyaluronidase $\bullet \mathrm{rHuPH} 20$-facilitated subcutaneous infusion of IG $\bullet$ subcutaneous administration of immunoglobulin

\section{Immunoglobulin replacement therapy in primary immunodeficiency disease}

Comprising approximately 300 genetically transmitted immune system disorders, primary immunodeficiency diseases (PIDDs) are often associated with impaired production of protective antibodies that predispose patients to recurrent and potentially life-threatening bacterial infections [1-4]. Although the prevalence of PIDD is not accurately known, it is likely more common than generally believed. In 2013, extrapolation of data from global registries and epidemiologic surveys suggested that an estimated 6,000,000 individuals worldwide may have a PIDD [5]. In the United States, claims data showed that the prevalence of any PIDD diagnosis increased during 2001-2007 from 38.9 to 50.5 per 100,000 in privately insured patients and from 28.2 to 39.0 per 100,000 in publicly insured patients [6].

For $>60$ years, immunoglobulin $\mathrm{G}(\mathrm{IgG})$ replacement therapy has been the standard of care for treatment of antibody deficiency $[4,7]$. Immunoglobulin replacement therapy was first reported in 1952 when an 8-year-old boy with agammaglobulinemia and a 3.5 year history of serious infections was successfully treated with monthly subcutaneous infusions of human immune serum globulin [8]. In the 1950s, ' 60 s and '70s, intramuscular injections of $\mathrm{IgG}$ were the predominant mode of administration; however, they were associated with long-lasting pain at the injection site, hypotension, loss of consciousness, chest tightness, dyspnea and episodes of facial swelling in nearly $20 \%$ of the patients [9]. In addition, intramuscular injections were difficult in pediatric patients with little muscle mass, resulting in more frequent injections. Furthermore, adherence was poor due to the discomfort associated with the injections. In the 1980s, intravenous infusion (intravenously administered IgG [IVIG]) became the predominant mode of administration because it avoided the intense pain of injections and allowed for delivery of larger doses [7]. The recommended dose of IVIG was increased from $100 \mathrm{mg} / \mathrm{kg} / \mathrm{month}$ to $>400 \mathrm{mg} / \mathrm{kg} / \mathrm{month}$ after it was shown that higher trough IgG concentrations improved pulmonary outcomes [10-12]. Although treatment with IVIG is 
effective in preventing infections, it is also associated with headache, fever, allergic reactions and other systemic reactions [13]. In addition, IVIG replacement therapy takes a toll on patients' quality of life because it requires repeated venous access and time-consuming administration in physician offices, outpatient infusion centers or scheduling with a home infusion nurse. Subcutaneously administered IgG (SCIG), which became available in 2006 [14], has been widely adopted clinically because unlike IVIG, it may be self-administered and is particularly attractive to patients with limited venous access. Survey data of patients and caregivers showed they preferred selfadministration versus administration by a healthcare professional and infusions at home rather than in a hospital or outpatient setting $(\mathrm{p}<0.05)$ [15]. Compared with IVIG, SCIG also has fewer systemic adverse events (AEs). As expected, some patients treated with SCIG experience some local pain, itching, erythema, edema and induration at the injection site; however, these reactions are usually mild, resolve within $24-48 \mathrm{~h}$, and decrease in frequency and severity as the therapy continues. However, limitations in the volume that can be infused into subcutaneous tissue with conventional SCIG requires frequent administration (i.e., weekly or biweekly) and multiple infusion sites $[7,13]$.

One strategy that effectively overcomes many of the limitations of conventional SCIG involves preceding the subcutaneous infusion of $\mathrm{IgG}$ with an infusion of hyaluronidase, an enzyme that catalyzes depolymerization of hyaluronan, resulting in enhanced drug delivery by increasing dispersion and absorption of IgG from the subcutaneous tissues, allowing larger volumes to be infused in each infusion site [16]. Recombinant human hyaluronidase (rHuPH20)-facilitated subcutaneous infusion of IgG (IGHy) is commercially available as HyQvia (Baxalta US, Inc., CA, USA, now part of Shire). This article reviews the use of $\mathrm{rHuPH} 20$ to facilitate subcutaneous infusions of $\mathrm{IgG}$ and provides an update on clinical data and practical applications of this therapeutic modality.

\section{Recombinant human hyaluronidase}

In 1929, Duran-Reynals described an enzymatic substance found in rabbit testes that enhanced the potency of intradermal injected virus [17]. This discovery led to the commercial development of bovine testes-derived hyaluronidase and its approval in 1948 for use as a spreading factor [18]. Today, hyaluronidase from bovine, ovine and recombinant-human sources is used clinically to increase the dispersion and absorption of subcutaneously administered drugs. However, animal-derived products are associated with IgE-mediated allergic reactions and are contaminated with proteases, immunoglobulins and factors that increase capillary permeability, all of which limit their use to short-term administration [19].

The naturally occurring glycosaminoglycan hyaluronan (or hyaluronic acid) is a key component of the gel-like ground substance of the extracellular matrix (ECM) in the connective tissue of the subcutaneous space. The ECM is a complex structure comprising collagen and elastin that maintains tissue structural integrity, glycosaminoglycans such as hyaluronan and other macromolecules. Hyaluronan is a large, viscous and hydroscopic molecule that impedes movement of fluids from the subcutaneous space to the vasculature. Unlike collagen and elastin and other stable structural components of the ECM, hyaluronan is short lived, with a half-life of 15-20 h [19].

The human PH2O gene was identified in the 1990s, leading to the development of rHuPH20 [18,20]. Using genetic recombinant technology, $\mathrm{rHuPH} 20$ was engineered using a construct encoding a soluble, neutral-active form of the human PH2O gene resulting in a soluble variant of the human PH20 enzyme that was highly specific (>100,000 United States Pharmacopeia units/mg protein) and 100-times more pure than bovine testes-derived hyaluronidase [19].

When injected into subcutaneous tissue, $\mathrm{rHuPH} 20$ temporarily depolymerizes hyaluronan, creating nanometersized microchannels that increase the permeability of the ECM, thereby enhancing the dispersion and absorption of infused fluids and drugs. The enzymatic activity of rHuPH20 is highly specific for the $\beta 1-4$ linkage in glycosaminoglycans, and it does not degrade structural protein components in the skin (e.g., collagen or elastin) or increase vascular permeability. $\mathrm{rHuPH} 20$ is suitable for long-term use because it is highly purified and not associated with inflammation, increased vascular permeability or frequent allergic reactions [19]. Use of rHuPH20 facilitates the infusion of large volumes of IgG into a single infusion site. The rHuPH20-facilitated infusion results in minimal swelling and the effects of $\mathrm{rHuPH} 20$ are completely reversible within $24-48 \mathrm{~h}$ (Figure 1). This is in contrast to conventional subcutaneous infusions, in which hyaluronan limits the permeability of the ECM, and the deliverable volume of drug that can be infused in a single site. The volume of conventional SCIG infusions has been limited to $20-60 \mathrm{ml} /$ site depending on the SCIG formulation [21,22], necessitating frequent infusions and the use of multiple infusion sites because infusion of larger volumes resulted in significant induration, reduced absorption of $\operatorname{IgG}$ and pain. 


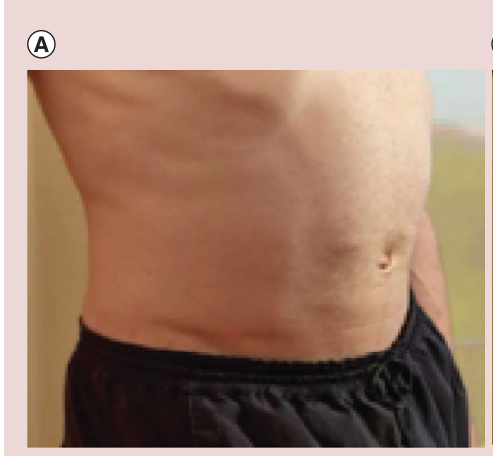

(B)

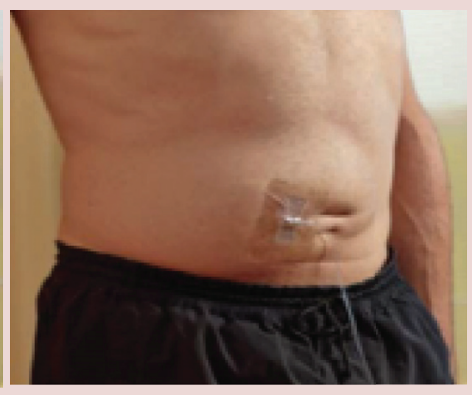

(c)

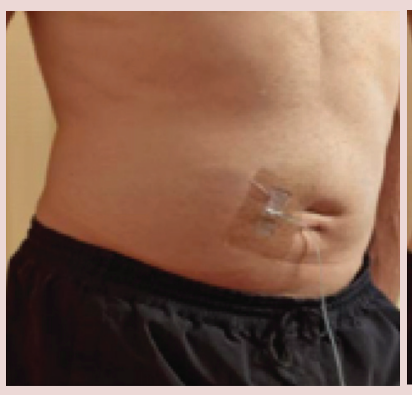

(D)

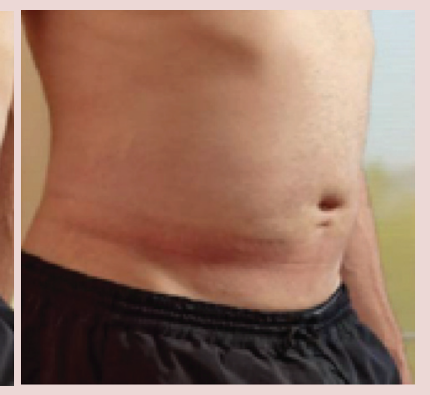

Figure 1. Subcutaneous infusion of $500 \mathrm{ml}$ of hyaluronidase-facilitated subcutaneous administration of immunoglobulin $\mathrm{G}$ using a single infusion site results in minimal and transient swelling.

(A) Before IGHy infusion; (B) after $300 \mathrm{ml}$ is infused; (C) after $500 \mathrm{ml}$ is infused; (D) $24 \mathrm{~h}$ after infusion.

Photographs courtesy of Shire US, Inc.

IGHy: Hyaluronidase-facilitated subcutaneous administration of immunoglobulin G.

Preclinical studies have demonstrated that $\mathrm{rHuPH} 20$ is fast acting and safe. In a mouse model, $\mathrm{rHuPH} 20$ had a local half-life in the subcutaneous space of $<30 \mathrm{~min}$, and in a rat model, $\mathrm{rHuPH} 20$ was detectable in the blood only at the $1 \mathrm{~min}$ time point following intravenous administration [18,23]. In primates, subcutaneous administration of rHuPH20 was well-tolerated at >9000-times the typical dose given with IGHy (45,000 units/injection) with no detectable inflammatory reactions or histologically identifiable inflammatory cell infiltrates compared with the carrier control from $24 \mathrm{~h}$ to 28 days postinjection [18]. Data from swine demonstrated that $\mathrm{rHuPH} 20$ enabled subcutaneous administration of large $\operatorname{IgG}$ volumes $(300 \mathrm{ml})$ at a single site. $\mathrm{rHuPH} 20$ minimized local tissue damage, significantly improved $\operatorname{IgG}$ dispersion (reduced mean local swelling volume and area) and reduced mean inline pressure associated with IgG infusion [24].

There have been many reports of the clinical immunogenicity of recombinant human proteins [25]. Interestingly, the prevalence of pre-existing anti-rHuPH 20 antibodies in healthy plasma donors has been determined to be approximately $6 \%$. Therefore, it is not unexpected that anti-rHuPH20 antibodies are present in a small percentage of patients before exposure to rHuPH20. It is not known why anti-rHuPH20 antibodies are present in some untreated and otherwise healthy individuals, but it may be related to hyaluronidase activity in sperm [26]. PH20 is expressed in the adult male reproductive tract and on sperm. There is no expression of PH20 in females at any stage of life in any tissue or in prepubertal males. In mammalian fertilization, hyaluronidase is released by the sperm to digest the hyaluronan in the zona pellucida, playing a nonobligate role in fertilization. Theoretically, a neutralizing antibody to rHuPH20 could cross-react with human PH20, inhibiting this activity. However, studies of $\mathrm{PH} 20$ knockout mice immunocontraceptive trials with anti-PH20 antibodies in mice and rabbits have shown that it plays a nonessential role in fertility [27-30]. Large doses of $\mathrm{rHuPH} 20$, such as those used to elicit high titers of neutralizing anti-rHuPH20 antibodies in animal studies, did not result in an observable effect on fertility, reproduction, fetal development or postnatal life [31]. Preliminary findings from a large, ongoing general population study of 692 adult men and women who answered a questionnaire covering selected medical and reproductive history and submitted a blood sample have not identified a relationship between infertility and the presence of rHuPH20-binding antibodies [32].

rHuPH20 is used to improve the dispersion and absorption of SCIG and other drugs (Table 1) [33]. Rituximab and trastuzumab are monoclonal antibodies used in the treatment of lymphoma and breast cancer, respectively. Monoclonal antibodies are typically administered intravenously over the course of hours, but subcutaneous infusions have been shown to reduce infusion times. The subcutaneous route, however, is limited by the volume needed to achieve plasma concentrations equivalent to intravenous administration. Infusion of $\mathrm{rHuPH} 20$ before subcutaneous administration of rituximab and trastuzumab has been shown to be safe and to result in plasma concentrations similar to intravenous administration, but with markedly shorter infusion times [34,35]. For example, pretreatment with $\mathrm{rHuPH} 20$ reduced rituximab administration from $\geq 1.5 \mathrm{~h}$ for intravenous infusion time to $5-7$ min subcutaneously [36]. 


\begin{tabular}{|c|c|c|c|c|}
\hline rHuPH20 \pm Product & Product name & Therapeutic area & Use/indication & Development stage \\
\hline rHuPH20 & 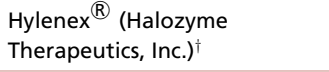 & Various; endocrinology & $\begin{array}{l}\text { Peptide, small molecule and } \\
\text { fluid delivery }\end{array}$ & Approved (FDA) \\
\hline rHuPH20 + IgG 10\%‡ (IGHy) & HyQvia (Baxalta, Inc.) $)^{\S}$ & Immunology & Primary immunodeficiency & Approved (EMA and FDA) \\
\hline rHuPH20 + trastuzumab & $\begin{array}{l}\text { Herceptin }{ }^{\circledR} \text { SC (Roche Pharma } \\
\text { AG) }\end{array}$ & Oncology & Breast cancer & Approved (EMA), Phase III (FDA) \\
\hline rHuPH20 + rituximab & $\begin{array}{l}\text { MabThera }{ }^{\circledR} \text { SC (EU) and } \\
\text { Rituxan SC (USA) (Roche } \\
\text { Products) }\end{array}$ & Oncology & Non-Hodgkin lymphoma & Approved (EMA and FDA) \\
\hline rHuPH20 + insulin & Analog Insulin-PH20 & Endocrinology & Diabetes & Phase II \\
\hline PEGylated rHuPH20 & $\begin{array}{l}\text { PEGPH20 (Halozyme } \\
\text { Therapeutics, Inc.) }\end{array}$ & Oncology & Solid tumors & Approved (EMA), Phase III (FDA) \\
\hline \multicolumn{5}{|c|}{ 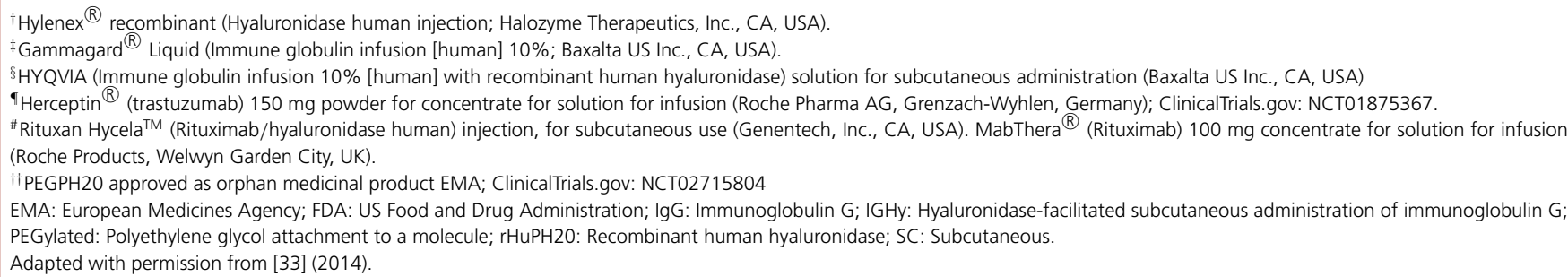 } \\
\hline
\end{tabular}

\section{Clinical studies of subcutaneously administered immunoglobulin G facilitated with recombinant human hyaluronidase in patients with primary immunodeficiency disease Phase I/II study}

The results from a Phase I/II study (ClinicalTrials.gov identifier: NCT00782106; Sponsored by Baxalta, Inc., now part of Shire) demonstrated that IGHy enabled single-site subcutaneous infusion of monthly doses of 255-612 ml of $10 \% \operatorname{IgG}(25.5-61.2 \mathrm{~g})$ at rates of $120-300 \mathrm{ml} / \mathrm{h}$, with bioavailability similar to IVIG [37]. Eleven patients with PIDD were enrolled and received IGHy administered subcutaneously using an infusion pump similar to one used to administer IVIG. A minimum dose of $50 \mathrm{U} \mathrm{rHuPH} 20 / \mathrm{g} \mathrm{IgG}$ was needed to enable rapid infusion of several hundred milliliters of IgG. Doses $\leq 300 \mathrm{U}$ rHuPH20/g IgG were evaluated, but $>50 \mathrm{U} \mathrm{rHuPH} 20 / \mathrm{g}$ IgG did not result in faster infusion rates or improved tolerability. Ten of the 11 patients completed the study; 1 patient withdrew because of moderate infusion-site discomfort after receiving $25 \%$ of the monthly dose of $\operatorname{IgG}$ administered subcutaneously with $\mathrm{rHuPH} 20$. Infusion rates depended on pump capacity, not patient tolerance. Eight of 10 patients achieved an infusion rate of $300 \mathrm{ml} / \mathrm{h}$. The mean infusion duration of $2.9 \mathrm{~h}( \pm 0.8 \mathrm{~h})$ was comparable to monthly intravenous infusions or weekly subcutaneous infusions [37]. AEs comprised mild swelling or redness at the infusion site. Allergic reactions were not reported [37].

Phase III \& extension studies

Study design \& patients

Based on the findings of the Phase I/II study [37], a Phase III prospective, open-label study was conducted to determine the efficacy, safety, tolerability and pharmacokinetics of IGHy in patients with PIDD (NCT00814320; Sponsored by Baxalta, Inc, now part of Shire). The primary end point was the annual rate of acute serious bacterial infections [16]. In this study, 87 adults and children 4-78 years of age with PIDD received IVIG every 3 or 4 weeks for 3 months, and 83 patients were then treated with IGHy for approximately 14-18 months. Of the 83 patients, 31 received 3 months of IVIG followed by 12 months of conventional SCIG without rHuPH20 in a previous study (NCT00546871) [38] and then were enrolled to receive IGHy for 14-18 months in the Phase III study [16].

All patients received IGHy at the same dose and frequency as their prestudy IVIG. Patients received rHuPH20 at a dose of $75 \mathrm{U} / \mathrm{g} \mathrm{IgG} \mathrm{administered} \mathrm{through} \mathrm{a} \mathrm{24-gauge} \mathrm{high-flow} \mathrm{subcutaneous} \mathrm{needle} \mathrm{followed} \mathrm{by} \operatorname{IgG} 10 \%$ at $108 \%$ of the weekly IVIG equivalent dose, using the same subcutaneous needle and infusion line [16]. The 108\% dose of IgG was based on the finding from the Phase I/II study that the bioavailability of IGHy was $92 \%$ of the IVIG dose [37]. In order to help patients and their infusion nurses psychologically adapt to the larger infusion volumes possible with IGHy and to provide clustered teaching opportunities, treatment was ramped up beginning with a fourth (for patients on a 4-week schedule) or a third (for patients on a 3-week schedule) of the monthly dose. 
Table 2. Recombinant human hyaluronidase-facilitated subcutaneous infusion of Immunoglobulin $\mathrm{G}$ infusions during pivotal (excluding ramp-up) and extension studies by age group $(<18, \geq 18$ years).

\begin{tabular}{|c|c|c|c|c|}
\hline \multirow[t]{2}{*}{ Age group, years ${ }^{\dagger}$} & \multicolumn{2}{|c|}{ Pivotal study (Excluding ramp-up) } & \multicolumn{2}{|c|}{ Extension study } \\
\hline & Patients treated, $n$ & Infusions, $n$ & Patients treated, $n$ & Infusions, $n$ \\
\hline$<18$ & 22 & 310 & 15 & 364 \\
\hline$\geq 18$ & 59 & 819 & 48 & 1236 \\
\hline Total & 81 & 1129 & 63 & 1600 \\
\hline
\end{tabular}

\section{Table 3. Duration of infusions.}

\begin{tabular}{|c|c|c|c|}
\hline Treatment & Age group, years & Patients, $\mathrm{n}$ & Duration of infusion, $h$, median (range) \\
\hline \multirow[t]{3}{*}{ IVIG } & $2-<12$ & 14 & $2.49(1.40-5.03)$ \\
\hline & $\geq 12$ & 73 & $2.33(0.92-6.33)$ \\
\hline & All patients & 87 & $2.33(0.92-6.33)$ \\
\hline \multirow[t]{3}{*}{ IGHy } & $2-<12$ & 11 & $1.73(1.15-3.28)$ \\
\hline & $\geq 12$ & 70 & $2.13(0.83-4.68)$ \\
\hline & All patients & 81 & $2.08(0.83-4.68)$ \\
\hline
\end{tabular}

IGHy: Recombinant human hyaluronidase-facilitated subcutaneous administration of immunoglobulin G; IVIG: Intravenously administered immunoglobulin G. Reproduced with permission from [16].

That dose was doubled 1 week later. Two weeks after that, three fourth of the monthly dose was administered to 4-week schedule patients while 3-week schedule patients received their full 3-weekly dose. That dose and interval was maintained for approximately 1 year [16].

Infusion rates $\leq 160 \mathrm{ml} / \mathrm{h}$ (patients $<40 \mathrm{~kg}$ ) and $\leq 300 \mathrm{ml} / \mathrm{h}(\geq 40 \mathrm{~kg}$ ) were allowed. Patients needing infusions of $>600 \mathrm{ml}$ had the option of using two infusion sites. After a training period, patients (or caregivers) self-administered the IGHy infusions at home [16]. During an extension study (NCT01175213; Sponsored by Baxalta, Inc, now part of Shire), the dose and interval of IGHy were maintained for the first three infusions, after which patients could switch from a 3-or 4-week dose/interval to a 2-week interval (with 50\% of the calculated 4-week dose) for $\leq 4$ months in order to determine trough levels for the 2-week interval [39]. A total of 66 patients who completed the Phase III study entered the long-term extension study; 63 patients continued on IGHy and 3 continued on IVIG [39].

\section{Infusion characteristics}

A total of 2729 infusions were administered during the Phase III study (1129, excluding ramp-up [initial 1-week dose of IGHy and increased until prestudy IVIG interval of 3 or 4 weeks was reached], and1600 in the extension study; Table 2) [39].

In the Phase III study, the median duration of IGHy infusions for all patients was $2.08 \mathrm{~h}$ (range: $0.83-4.68 \mathrm{~h}$; Table 3), which was comparable to the median duration of infusion during the IVIG portion of the study of $2.33 \mathrm{~h}$ (range: $0.92-6.33 \mathrm{~h}$ ) [16]. The median ratio of IGHy to intravenous was $108.8 \%$ (range: $98.6-138.2 \%$ ). The mean volume of IGHy per infusion site was $292.2 \mathrm{ml}$ overall in the Phase III study (excluding ramp-up), including mean volumes of $174.6 \mathrm{ml}$ for 2-week intervals, $276.5 \mathrm{ml}$ for 3-week intervals and $302.2 \mathrm{ml}$ for 4-week intervals [16]. In the Phase III (excluding ramp-up) and extension studies, mean \pm SD doses of IGHy were $0.155 \pm 0.053 \mathrm{~g} / \mathrm{kg} / \mathrm{week}$ and $0.156 \pm 0.051 \mathrm{~g} / \mathrm{kg} /$ week, and mean IGHy treatment duration was $367.7 \pm 103.9$ days and $565.9 \pm$ 211.8 days, respectively. A total of 48 patients were treated for $>30$ months [39].

\section{Pharmacokinetic properties}

As measured by the area under the concentration time curve, IGHy administered at $108 \%$ of the IVIG dose was bioequivalent to IVIG (US FDA definition of subcutaneous bioequivalence is $80-120 \%$ of intravenous dose); IGHy bioavailability was $93.3 \%$ (90\% CI: 91.4-95.2\%). A 20.4\% improvement in bioavailability was observed with IGHy relative to SCIG [16]. The slower IgG entrance into the intravascular space following IGHy infusion 


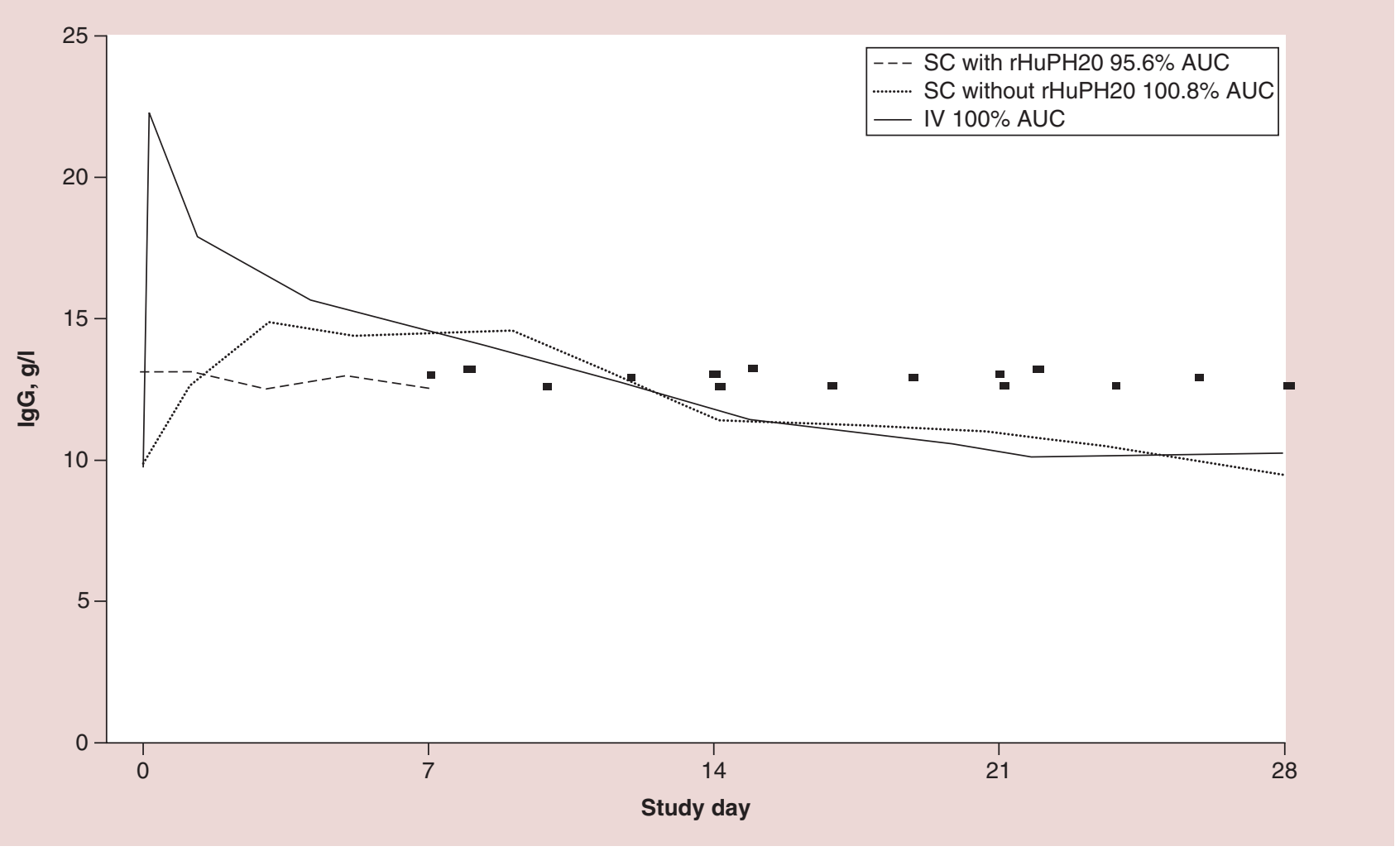

Figure 2. Serum immunoglobulin G concentration for recombinant human hyaluronidase-facilitated subcutaneously administered immunoglobulin G compared with intravenously administered immunoglobulin G and subcutaneously administered immunoglobulin G. Representative pharmacokinetic curves for a patient comparing a 4-week infusion of IVIG, a weekly infusion of SCIG, $10 \%$ at $143 \%$ of the intravenous dose with the same data points extended across a 4-week period to facilitate comparison with the other curves, and a 4-week infusion of IGHy at $104 \%$ of the intravenous dose.

AUC: Area under the curve; IgG: Immunoglobulin G; IGHy: Recombinant human hyaluronidase-facilitated subcutaneously administered immunoglobulin G; IVIG: Intravenously administered IgG; rHuPH20: Recombinant human hyaluronidase; SCIG: Subcutaneously administered IgG.

Reproduced with permission from [16].

was indicated by lower median peak serum IgG levels with IGHy $(15.5 \mathrm{~g} / \mathrm{l})$ compared with IVIG $(21.9 \mathrm{~g} / \mathrm{l})$ and a later median peak IgG concentration with IGHy ( 5 days after infusion with IGHy compared with 0.1 days with IVIG) [16]. When IGHy or IVIG was administered at 3- or 4-week intervals, median serum IgG trough levels were similar $(10.7 \mathrm{~g} / \mathrm{l}$ and $10.4 \mathrm{~g} / \mathrm{l}$, in patients aged $\geq 12$ years, respectively) regardless of the administration route. Figure 2 shows serum IgG concentration for IGHy compared with IVIG and SCIG for a representative patient [16]. The median IgG trough level during the SCIG portion of the study reflected the higher SCIG dose (1.37-times the IVIG dose) and the time after infusion that trough levels were obtained (1 week vs 3 or 4 weeks).

The appropriate trough to minimize infections is individual to each patient $[40,41]$. By protocol, the minimum $\mathrm{IgG}$ trough level in the study was $500 \mathrm{mg} / \mathrm{dl}$. The median steady-state IgG trough levels during IGHy infusions was $983 \mathrm{mg} / \mathrm{dl}$ (95\% CI: 946-1070) during 4-week intervals; it was higher for the at 3- and 2-week interval recipients (1195 mg/dl [95\% CI: 958-1530] and $1135 \mathrm{mg} / \mathrm{dl}$ [95\% CI: 939-1440], respectively) [16,39].

Population pharmacokinetic modeling using data from the Phase III and extension studies in previously IgGnaive patients suggested that the time to reach steady-state IgG trough levels with IGHy was similar to IVIG (18 vs 16 weeks, respectively). Simulated median trough concentrations during the IGHy ramp-up exceeded $700 \mathrm{mg} / \mathrm{dl}$ by the end of the ramp-up phase at 10 weeks after initiating treatment [42].

\section{Protection against bacterial infections}

During the Phase III and extension studies, there were five acute serious bacterial infections, all of which were pneumonias [39]. Overall, the rates of serious bacterial infections during IGHy treatment in the Phase III and 
extension studies were 0.025 (upper 99\% CI limit: 0.046) and 0.03 (upper 99\% CI limit: 0.05) per patient-year, respectively [16,39], which were substantially lower than the predefined rate threshold of $<1.0$ infection per personyear outlined by the FDA's Blood Products Advisory Committee for demonstration of efficacy of IgG replacement therapy [43]. The annual rate for all infections during IGHy exposure in the Phase III study was 2.97 (95\% CI: 2.51-3.47) per patient-year for IGHy and 4.51 (95\% CI: 3.50-5.69) for IVIG treatment (annual rate based on the 3 months of treatment), which is comparable to other modes of IgG treatment from other studies [16]. The overall annual infection rate for IGHy throughout both the Phase III and extension studies was 2.99 (95\% CI: 2.60-3.42). Infection rates over 1 year periods of IGHy treatment in the Phase III and extension studies remained stable, and the rates were comparable for children/adolescents and adults [39].

Sinusitis, upper respiratory tract infections, viral upper respiratory tract infections and bronchitis were the most common infections reported during IGHy treatment in the extension study [39].

Treatment with IGHy was associated with low rates of infection-associated adverse outcomes [16]. During IGHy treatment in the extension study, the annual rate of days missed from school or work was 5.75, unscheduled outpatient physician visits was 4.67 , hospitalization was 0.12 and days hospitalized was 0.61 . The rate of days receiving antibiotics per year, including brief infection prophylaxis for surgical or dental procedures was 65.39 [39].

\section{Safety \& tolerability}

In the Phase III study, the majority of AEs experienced during IGHy treatment were mild or moderate, and there were no drug-related serious AEs. The common AEs during IGHy treatment were local adverse reactions such as discomfort/pain (rate: 0.108 per infusion), erythema (rate: 0.028 per infusion) and swelling/edema (rate: 0.024 per infusion). The rates of causally related local AEs per IGHy infusion was 0.203 , which is comparable to rates observed with conventional subcutaneous administration of IgG, despite the larger infusion volume with IGHy. Rates of temporally associated local AEs per infusion (occurring $\leq 72 \mathrm{~h}$ after infusion) was 0.199 for IGHy [16].

During 187.7 patient-years of IGHy exposure, $2005 \mathrm{AEs}$ (excluding infections) were reported (10.68 per patientyear). Of the 498 local AEs (2.65 per patient-year), 488 (2.60 per patient-year) were considered treatment related and 488 (2.60 per patient-year) occurred during or $\leq 72 \mathrm{~h}$ after IGHy infusion. There were no local serious AEs. Local AEs, including infections, occurred at a rate of 0.17 per infusion [39].

The rate of related local AEs decreased from 3.68 per patient-year in the first year to 1.5 per patient-year after 30 months of treatment. Rates of local AEs for patients with a BMI $<25 \mathrm{~kg} / \mathrm{m}^{2}$ were 4.27 per patient-year $(0.13$ per infusion), compared with 7.47 per patient-year (0.19 per infusion) for patients with a BMI of $25-30 \mathrm{~kg} / \mathrm{m}^{2}$ and 9.33 per patient-year ( 0.23 per infusion) for patients with a BMI $>30 \mathrm{~kg} / \mathrm{m}^{2}$ [39].

Among the 1507 systemic AEs (8.03 per patient-year; excluding infections) reported during IGHy treatment, 329 (1.75 per patient-year) were considered related to IGHy treatment and 491 (2.62 per patient-year) occurred during or $\leq 72 \mathrm{~h}$ after treatment [39].

The most common AEs during IGHy treatment were headache ( 0.59 per patient-year; $54.2 \%$ of patients; $3.51 \%$ of infusions), nausea $(0.39 ; 32.5 \% ; 2.37 \%)$, vomiting $(0.20 ; 28.9 \% ; 1.28 \%)$, asthma $(0.32 ; 28.9 \% ; 1.89 \%)$, fatigue $(0.28 ; 26.5 \% ; 1.72 \%)$, infusion-site erythema $(0.37 ; 25.3 \% ; 2.37 \%)$, pyrexia $(0.22 ; 25.3 \% ; 1.35 \%)$ and diarrhea $(0.18 ; 24.1 \% ; 1.12 \%)$. Serious hypersensitivity reactions did not occur during long-term IGHy treatment [39].

\section{Pediatric patients}

Treatment of pediatric patients with IGHy in intervals of 3 or 4 weeks maintained protective trough levels, prevented infections and was well-tolerated [44]. A total of 26 patients aged $<18$ years were enrolled in the Phase III trial, of whom 24 received IGHy. Of these, 15 patients were included in the extension study and received IGHy treatment for a median of 2.46 years. During the Phase III and extension studies, the total exposure among pediatric patients was 48.7 patient-years, and the median number of infusions per patient was 33.5. The majority of infusions were completed without interruption or reduction of infusion rates due to AEs (97.2\% of 674 infusions, excluding ramp-up) and required only a single infusion site (82.5\% of 738, including ramp-up). Mean infusion volumes per site were $176 \mathrm{ml}$ for children 4 to $<12$ years of age (range: $22-313 \mathrm{ml}$ ) and $306 \mathrm{ml}$ for adolescents $\geq 12$ to 18 years of age (range: $41-616 \mathrm{ml}$ ). The median infusion duration was $1.65 \mathrm{~h}$ for IGHy, compared with $2.42 \mathrm{~h}$ for IVIG [44]. During the Phase III study, the median infusion duration was $1.73 \mathrm{~h}$ (range: 1.15-3.28) for children $<12$ years of age, compared with $2.13 \mathrm{~h}$ (range: $0.83-4.68$ ) for adolescents and adults ( $\geq 12$ to 18 years of age) [16].

During the Phase III and extension studies, the rate of acute serious bacterial infections per patient-year was 0.08 (upper limit of 99\% CI: 0.20) for pediatric patients. There were four cases of pneumonia in three patients. 


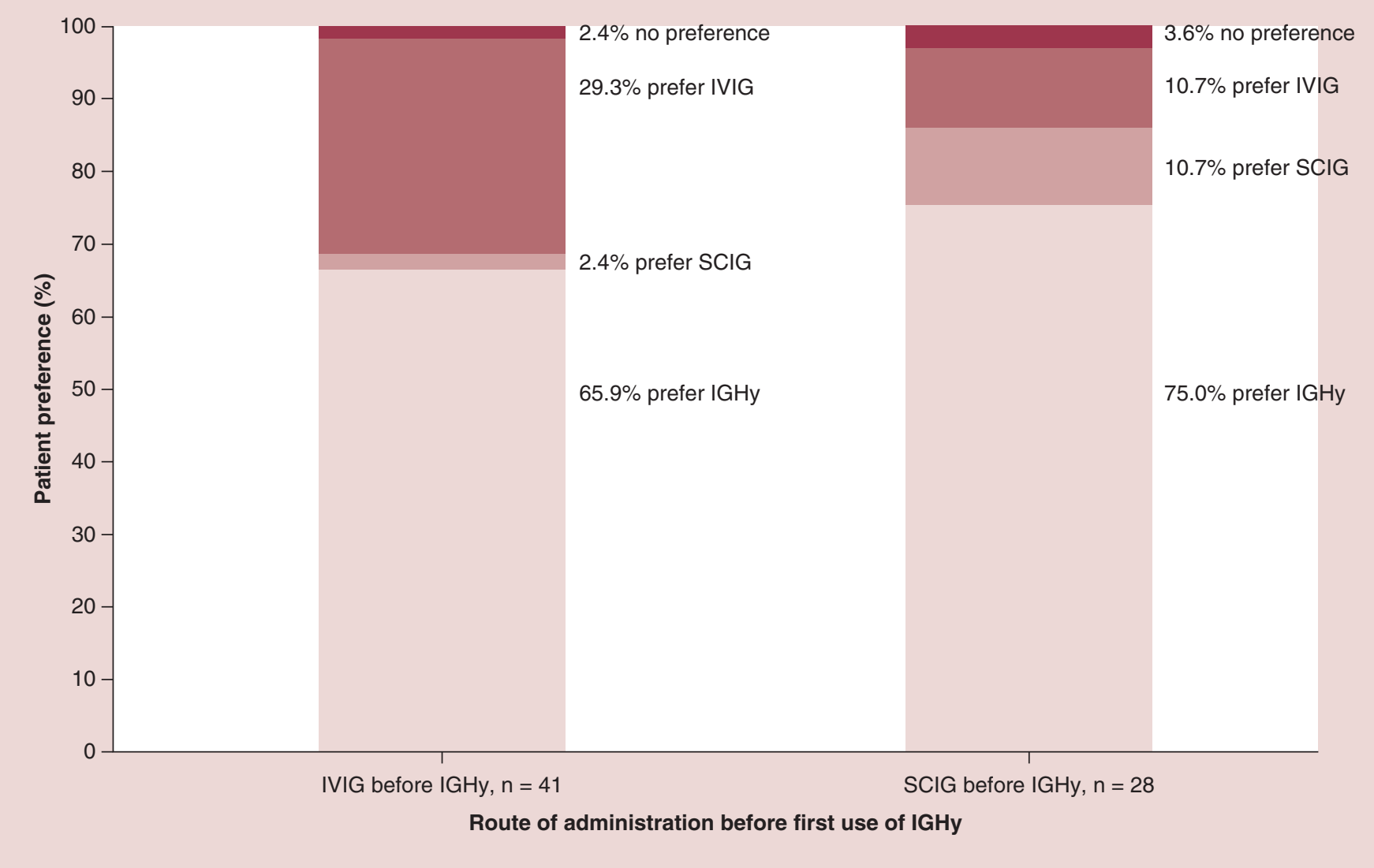

Figure 3. Patient preference for recombinant human hyaluronidase-facilitated subcutaneously administered immunoglobulin G, intravenously administered immunoglobulin G, or subcutaneously administered immunoglobulin G.

IgG: Immunoglobulin G; IGHy: Recombinant human hyaluronidase-facilitated subcutaneously administered immunoglobulin G; IVIG: Intravenously administered IgG; SCIG: Subcutaneously administered IgG.

Infection rates per patient-year were similar for patients $<18$ years of age $(3.02 ; 95 \% \mathrm{CI}: 2.15-4.10)$ and adults $\geq 18$ years of age $(2.98 ; 95 \%$ CI: $2.56-3.44)$ [44].

AEs in the pediatric population were mild or moderate in severity; six serious AEs were reported, but were not considered related to IGHy treatment [44]. Rates were low for causally related systemic $(0.10$ per infusion $)$ and local (0.09 per infusion) AEs. Headache (33.3\% of patients; 0.030 per infusion), pyrexia $(20.8 \%$; 0.019$)$ and fatigue $(16.7 \% ; 0.007)$ were the most commonly reported systemic reactions. Discomfort/pain ( 0.051 per infusion) and erythema (0.014 per infusion) were the most frequent local AEs. Swelling at the infusion site was transient and resolved in $24-48 \mathrm{~h}$. After 48.7 patient-years of IGHy exposure, no patient had clinically apparent changes in skin or subcutaneous tissue. Over the entire treatment period, ranging up to 3.3 years, rates of systemic events did not change; local AEs became less frequent [44].

\section{Patient preference}

A total of 69 patients completed a satisfaction survey at the completion of IGHy treatment. Based on the study design, one cohort of patients received IVIG then SCIG before receiving IGHy $(\mathrm{n}=28)$, and the second cohort only received IVIG before receiving IGHy $(\mathrm{n}=41)$. More than $65 \%$ of patients preferred treatment with IGHy (Figure 3); 65.9\% of patients (27 of 41) were treated with IVIG before their first use of IGHy and $75.0 \%$ of patients (21 of 28) treated with IVIG then SCIG before their first use of IGHy preferred IGHy [39]. All pediatric patients' caregivers who responded for patients aged $<14$ years preferred to continue IGHy treatment rather than switch to IVIG or conventional SCIG. For adolescent patients $\geq 14$ to $<18$ years of age, $85.7 \%$ preferred to continue IGHy treatment, and 1 patient (14.3\%) preferred to return to regular SCIG treatment [44]. 


\section{Anti-PH20 immunogenic response}

Using a highly sensitive binding assay, a total of $15(18 \%)$ patients transiently developed anti-rHuPH20 binding antibodies; 13 in the Phase III study and 2 in the extension study [16,39]. During the course of the studies, patients were monitored for development of $\mathrm{rHuPH} 20$-reactive binding or neutralizing antibodies and temporal relationships between seropositivity and AEs [39]. Before receiving IGHy in the Phase III study, all patients had been treated with pooled human IVIG, which contains low levels of $\mathrm{rHuPH} 20$-reactive antibodies. This passive antibody transfer resulted in pre-exposure, with baseline anti-rHuPH20 antibody titers ranging from 1:10 to 1:80. To account for the presence of these antibodies at baseline, the cutoff for anti-rHuPH20 antibody positivity was defined as titers $\geq 1: 160$. One patient had a positive baseline $\mathrm{rHuPH} 20$ antibody titer in the Phase III study and had previously been exposed to $\mathrm{rHuPH} 20$ in an earlier trial in which immunogenicity was not monitored [26].

No patient in the study developed neutralizing antibodies to hyaluronidase during or after exposure to IGHy [26,39]. Of the 13 patients who developed anti-rHuPH20 binding antibodies during rHuPH20 treatment in the Phase III study, 11 patients continued into the extension study and 6 continued to have titers $\geq 1: 160$. An additional 2 patients developed a 1:160 titer during the extension study, for an overall rate of 18\% (15 of 83 patients). Longitudinal data from 5 patients with titers $>1: 160$ demonstrated that titers decreased over time despite continued exposure to IGHy (Figure 4) [26,39]. Three of 24 pediatric patients (aged 13, 14 and 17 years) developed transient anti-rHuPH20 titers ( $\geq 1: 160)$ that decreased over time with continued IGHy treatment [44]. Isotyping of anti-rHuPH20 antibodies showed similar ratios of IgM, IgA and IgG (isotypes IgG1, IgG2, IgG3 and IgG4) for treatment-emergent antibodies from patients in the Phase III study, compared with rHuPH20 antibodies in a baseline-positive population with pre-existing rHuPH20 antibodies (Figure 5) [26]. Cross-reactivity immunoassays of anti-rHuPH20 antibodies and endogenous PH20 (e.g., human sperm-derived hyaluronidase) showed that IGHy does not cross-react with PH20 analogs. Indeed, there was no temporal association between the incidence or severity of systemic or local adverse reactions and the presence of anti-rHuPH20 antibodies [26,39]. Thus, findings from these studies show that IGHy treatment-emergent anti-rHuPH20 antibodies in patients with PIDD are non-neutralizing and have no observable clinical consequences. In fact, a double-blind, placebo- and within-patient, controlled study showed that single intradermal doses of $\mathrm{rHuPH} 20$ were well-tolerated with no evidence of allergenicity or immunogenicity [45].

\section{Real-world use of hyaluronidase-facilitated subcutaneous administration of immunoglobulin G}

To optimize the clinical outcome in patients with PIDD, the heterogeneity of diagnoses and the unique dose, dosing interval and IgG trough levels required must be considered. Immunoglobulin replacement therapy must therefore be tailored for each individual patient [46]. Subcutaneous infusion with IGHy provides options for individualized treatment that minimizes the impact of IgG replacement therapy on patients' lives. One of the benefits of IGHy treatment is that patients may choose to infuse larger volumes, thereby lengthening the dosing interval and minimizing the number of needle sticks. Data from the Phase III study of IGHy show that an infusion volume $\leq 600 \mathrm{ml}$ per site was well-tolerated [16]. A prospective, noninterventional, open-label, multicenter postauthorization safety study is ongoing to assess the long-term effects of IGHy in adult patients aged $\geq 16$ years in a routine clinical setting.

Factors contributing to treatment adoption and retention were examined in a series of 50 patients with PIDD from a single-practice cohort who were treatment naive $(n=7)$ or who transitioned from IVIG $(n=4)$ or conventional SCIG $(n=39)$ to IGHy [47]. Patients transitioning from IVIG cited poor venous access $(n=3)$ or the desire to self-infuse $(\mathrm{n}=1)$. Reasons patients chose to switch from SCIG to IGHy included less frequent infusions $(\mathrm{n}=30)$, fewer needle sticks $(\mathrm{n}=7)$ and AEs $(\mathrm{n}=2)$. Overall, $76 \%$ of patients (38 of 50$)$ remained on IGHy. Of the 39 patients who switched from SCIG, $85 \%(n=33)$ remained on IGHy. The majority of patients $(94 \%$; 47 of 50) infused IGHy every 4 weeks, and 8 changed the dosing interval from 4 to 3 weeks (to improve clinical response; $\mathrm{n}=5$ ) or from 3 to 4 weeks (because of positive response; $\mathrm{n}=1$ ) or to decrease the infusion volume/site $(\mathrm{n}=2)$. Thirty-one patients $(62 \%)$ initiated IGHy with one infusion site, and 25 of 31 patients $(80.6 \%)$ continued with a single site after dose ramp-up. Seven patients increased their infusion sites after completing dose ramp-up to decrease infusion volume per site; six patients switched from 1 to 2 sites, and one patient switched from 2 to 4 sites. The mean infusion volume per site was $233 \mathrm{ml}$ (range: $75-400 \mathrm{ml}$ ). Properly trained healthcare providers were best suited to educate patients and manage their expectations about the appearance of the postinfusion site, techniques for optimizing infusions and potential AEs. Patients should understand that they have the option to 
(A)

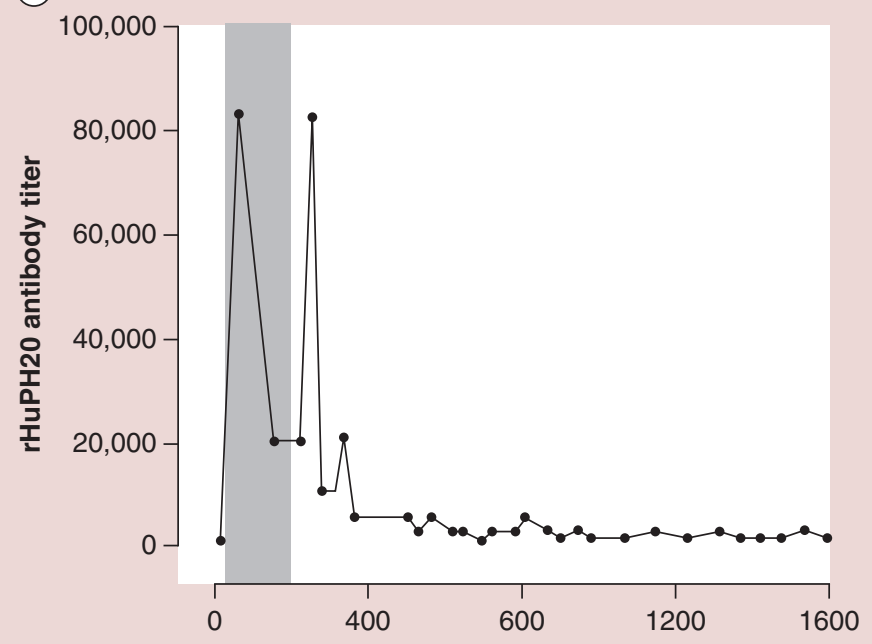

(C)

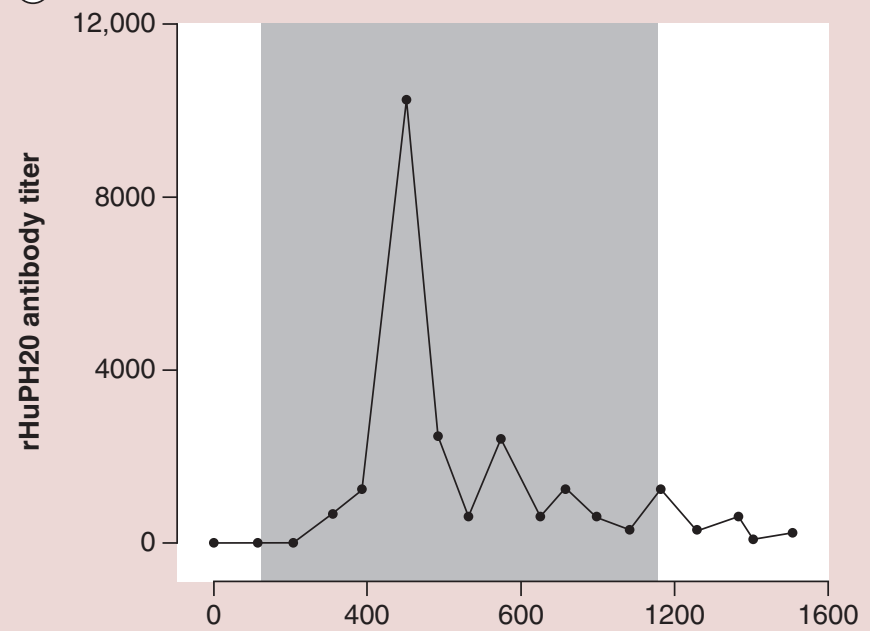

(E)

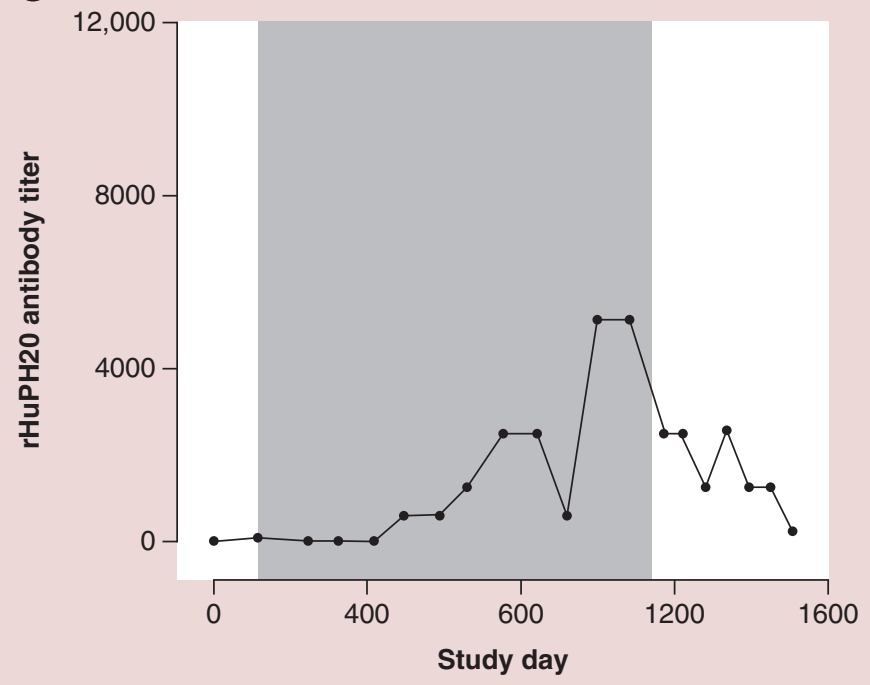

(B)

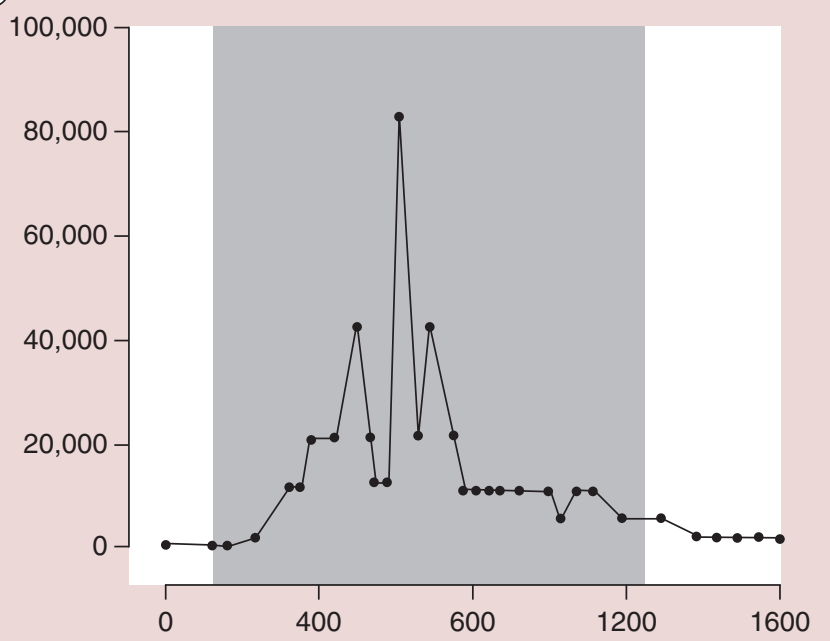

(D)

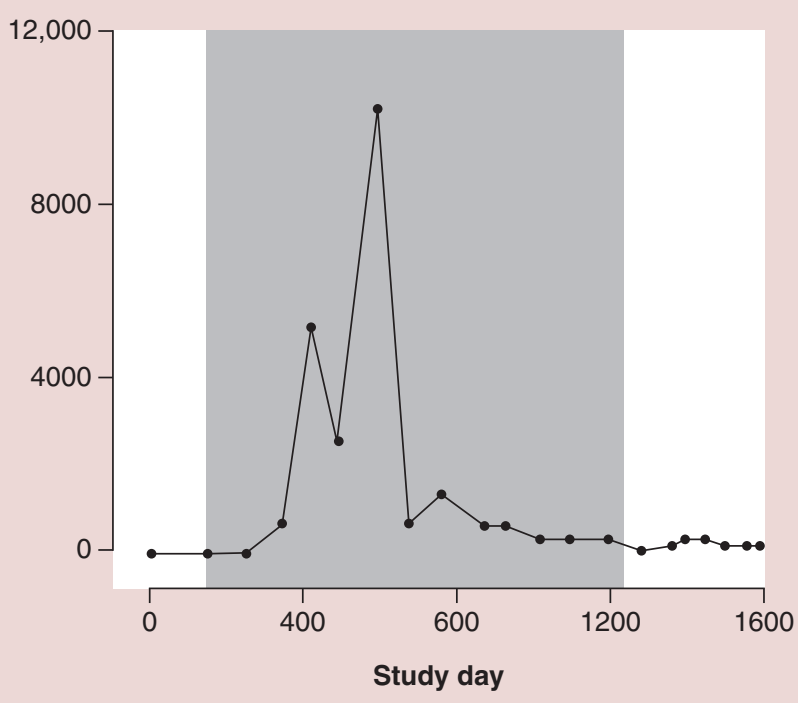

Figure 4. Recombinant human hyaluronidase-binding antibody titers over time for five patients (A-E) who had maximum titers $>1: 5000$. The titers of the single patient with a positive titer (1:160) before study participation are shown in (A).

Shaded areas indicate the period of rHuPH20 exposure.

rHuPH20: Recombinant human hyaluronidase.

Reproduced with permission from [26]. 
(A)

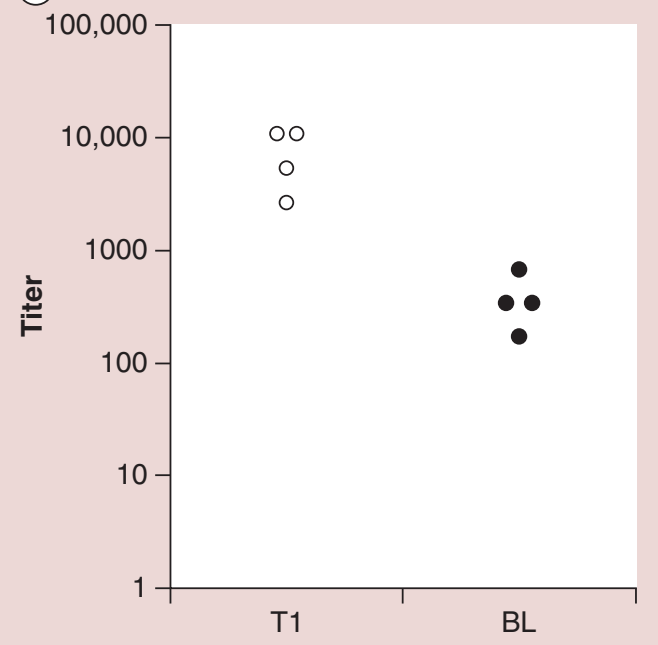

(C)

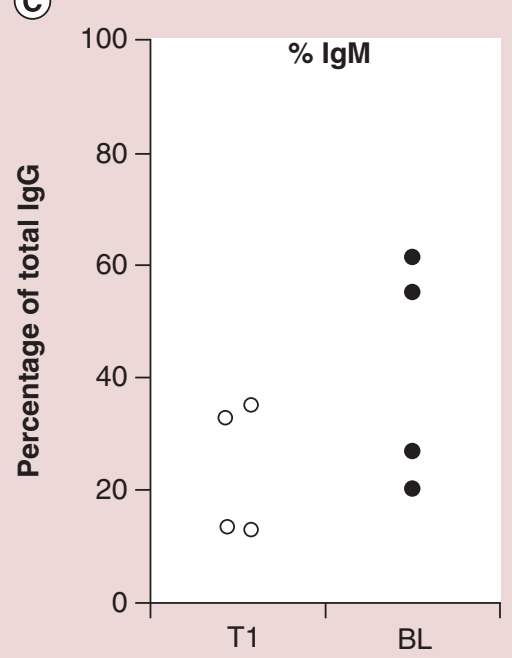

(D)

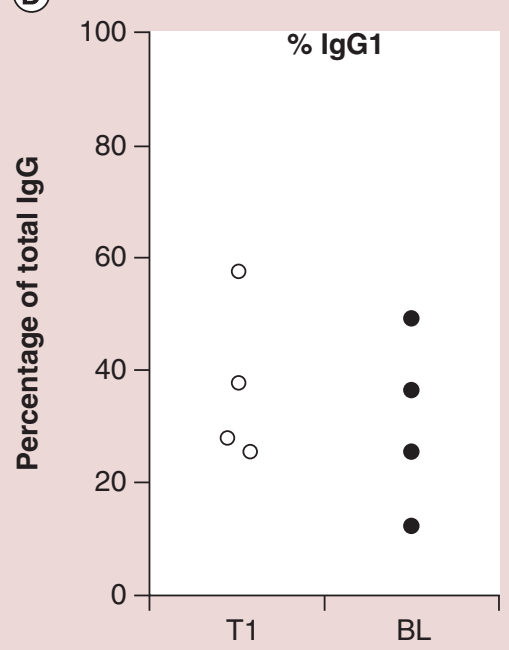

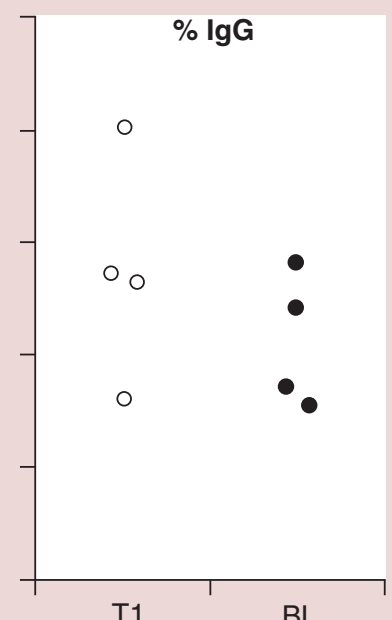

$\mathrm{BL}$

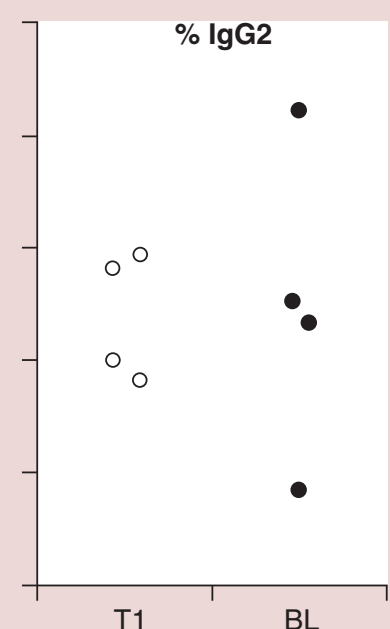

(B)

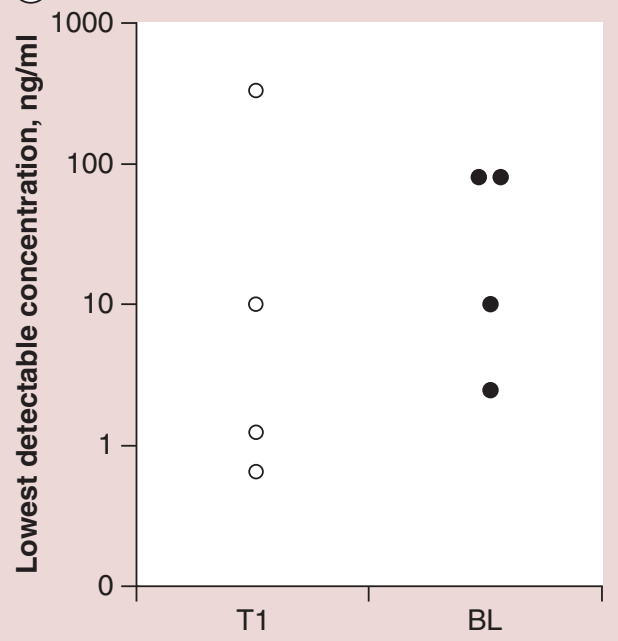

$\mathrm{T} 1$

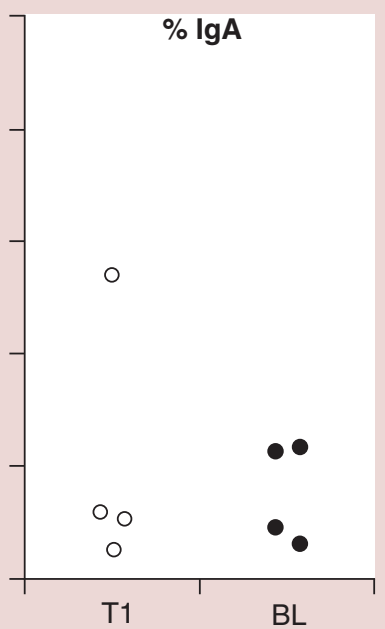

$\mathrm{BL}$
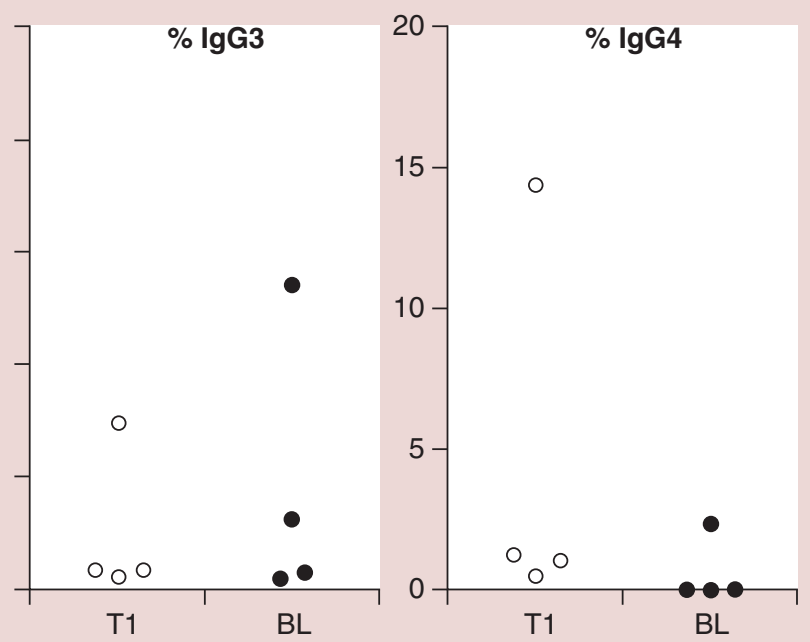

Figure 5. Recombinant human hyaluronidase-reactive antibodies from patients with positive antibody titers following exposure to recombinant human hyaluronidase (treatment-induced) and from the baseline-positive population with pre-existing recombinant human hyaluronidase antibodies (baseline). (A) Plasma titers at the time of antibody purification. (B) Lowest detectable concentration of purified rHuPH20-reactive antibodies. (C) Isotypes. (D) IgG subclasses. Observations from individual antibody preparations from four individuals are indicated.

BL: Baseline; IgG: Immunoglobulin G; rHuPH20: Recombinant human hyaluronidase; TI: Treatment-induced.

Reproduced with permission from [26]. 
change the number and location of infusion sites, increase dosing frequency and use longer needles to optimize their patient experience and treatment continuation [47].

Local AEs that may occur during subcutaneous IgG administration include swelling, erythema, discomfort, pruritus and infusion-site leakage $[16,48]$. Use of longer needles may affect local tolerability and prevent IgG leakage from the infusion site [48]. Needles used in subcutaneous infusions should be sufficiently long to penetrate deeply into subcutaneous tissue and avoid the local irritation that may be caused by shorter needles [48]. The choice of needle length for IGHy administration is based on clinical judgment.

Data on patients treated with IGHy through a specialty pharmacy were assessed for a relationship between needle length and BMI [48]. After the ramp-up period, 65 patients (58\%) chose one infusion site and 47 patients (42\%) preferred two infusion sites. Most patients (88 of 112; 79\%) infused IGHy with a $9 \mathrm{~mm}$ needle. However, $17 \%$ of patients switched to a $12 \mathrm{~mm}$ needle, primarily because of IgG leakage from the infusion site. Patients with higher BMIs tended to use $12 \mathrm{~mm}$ needles (mean BMI: $36.7 \mathrm{~kg} / \mathrm{m}^{2}$ ) compared with the $9 \mathrm{~mm}$ needles often used by patients with lower BMIs (mean BMI: $28.6 \mathrm{~kg} / \mathrm{m}^{2}$ ) [48].

A retrospective review of the real-world experience with IGHy in 13 pediatric patients 7-17 years of age showed that there was no correlation with BMI and needle length, and $92 \%$ of children used the $9 \mathrm{~mm}$ needle, a $24 \mathrm{G}$ high-flow needle set that is most commonly used in adults. The median volume/infusion site was $200 \mathrm{ml}$ (range: $100-300 \mathrm{ml}$ ), and doses ranged from 10 to $30 \mathrm{~g}$ per infusion every 3 to 4 weeks. The majority (70\%) of patients used one infusion site, and 30\% used two sites [49]. These data suggest that patient training sessions during dose ramp-up may help determine the ideal needle length and the number of infusion sites for each patient. Healthcare professionals and patients should discuss the comfort of infusion volumes, the number of infusion sites and the needle length that best suits each individual.

\section{Future perspective}

Immunoglobulin replacement therapy for PIDD has evolved considerably over the past 65 years from its inception as subcutaneous infusion in 1952 [8]. The subsequent stage in replacement therapy, intramuscular injections of IgG, used from the 1950s through the 1970s, was associated with significant and persistent injection-site pain that limited the dose as well as systemic reactions. The availability of more purified and better stabilized immunoglobulin products shifted the standard of practice in the United States and much of Europe to IVIG in the early 1980s, but systemic reactions and the need for repeated venous access and participation of a skilled healthcare provider rendered IVIG far from ideal for a disease requiring lifelong treatment [7]. The availability of IgG products for subcutaneous administration over the past 10 years has provided an alternative to IVIG therapy for patients who have difficulty with venous access, do not tolerate intravenous infusion, or prefer the ability to administer treatment in a home setting. However, because hyaluronan impedes the flow of fluid in the ECM, the amount of IgG that can be administered subcutaneously is less than that which can be administered intravenously - resulting in administration of small-volume doses in multiple infusion sites more frequently than typical IVIG treatment [33].

The availability of SCIG facilitated with $\mathrm{rHuPH} 20$ marked a significant milestone in the care of patients with PIDD [18,19]. This treatment provides a bioequivalent exposure to IgG and a 3- or 4-week dosing interval similar to those achieved with IVIG, but without the need for intravenous access required for repeated intravenous infusions. The use of IGHy is also preferred by many patients and caregivers because it can be administered at home with fewer needle sticks and larger infusion volumes than are possible with SCIG [7]. The management of PIDD will continue to improve as clinicians and patients gain experience with IGHy therapy. Real-world data are emerging that are demonstrating that healthcare provider training and patient education are critical to improving adherence, optimizing clinical outcomes and fostering an environment where patients and their caregivers can individualize treatment $[47,48]$.

The treatment paradigm for IgG replacement therapy increasingly emphasizes individualization of care. As such, a patient's therapy should be individualized to best meet their needs. IGHy may be a suitable treatment option for patients with PIDD who wish to reduce the burden of care by enabling relatively infrequent (every $2-4$ weeks) self-administered treatment that eliminates the need for visiting an infusion center and increasing infection risk [50] or scheduling a home visit with a specialty pharmacy nurse. As more physicians who care for patients with PIDD become familiar with IGHy, its use will increase. Subcutaneous infusion of IgG is safer and more convenient than intravenous infusion; IGHy is an important addition to the immunologist's armamentarium. 
Acknowledgements

Scientific writing was provided by SK Laden, MS and LM Klumpp Callan, PhD, of C4 MedSolutions, LLC (Yardley, PA), a CHC Group company. This assistance was funded by Shire, MA, USA.

Financial \& competing interests disclosure

RL Wasserman is an investigator for Bio Products Laboratory, Octapharma, Prometic, Shire, and Therapure, and is a consultant for Bio Products Laboratory, Grifols, Korean Green Cross, Prometic, Secretory IgA, Shire, and Therapure. The author is a speaker for Bio Products Laboratory and Shire. The author has no other relevant affiliations or financial involvement with any organization or entity with a financial interest in or financial conflict with the subject matter or materials discussed in the manuscript apart from those disclosed.

Writing assistance was utilized in the production of this manuscript. Scientific writing was provided by SK Laden, MS and LM Klumpp Callan, PhD, of C4 MedSolutions, LLC (Yardley, PA), a CHC Group company. This assistance was funded by Shire, MA, USA.

\section{Open access}

This work is licensed under the Attribution-NonCommercial-NoDerivatives 4.0 Unported License. To view a copy of this license, visit http://creativecommons.org/licenses/by-nc-nd/4.0/

\section{Executive summary}

Immunoglobulin replacement therapy in primary immunodeficiency diseases

- Primary immunodeficiency diseases (PIDDs) are a heterogeneous group of diseases associated with severe, recurrent bacterial infections.

- Immunoglobulin G (IgG) replacement therapy administered via intravenous (intravenously administered IgG [IVIG]) or subcutaneous (subcutaneously administered IgG [SCIG]) infusions is the standard of care for PIDD.

- IVIG requires venous access, typically employs supervised administration in outpatient settings and has a significant rate of systemic adverse events.

- SCIG has fewer systemic adverse events than IVIG and may be self-administered at home. However, because the extracellular matrix in subcutaneous tissue restricts fluid flow to the vascular space, there is a limit to the dose of IgG that can be infused in a single site, requiring frequent dosing intervals and multiple infusion sites.

Recombinant human hyaluronidase

- Hyaluronan is a large molecule found in the extracellular matrix that impedes fluid movement in the subcutaneous space.

- Hyaluronidase temporarily and reversibly degrades hyaluronan and is used to increase dispersion and absorption of subcutaneously administered drugs and fluids.

- Animal-derived hyaluronidase is immunogenic and causes allergic reactions.

- Recombinant human hyaluronidase ( $\mathrm{rHuPH} 20)$ safely and effectively improves the dispersion and absorption of SCIG and other drugs.

Clinical studies of subcutaneously administered immunoglobulin $\mathrm{G}$ facilitated by recombinant human hyaluronidase in patients with primary immunodeficiency diseases

- A Phase III study and extension study of hyaluronidase-facilitated subcutaneous administration of IgG (IGHy) in 83 adults and pediatric patients with PIDD demonstrated that IGHy was typically infused every 3 to 4 weeks over the course of $2.1 \mathrm{~h}$, was often completed with a single infusion site and may be self-administered.

- Rates of acute serious bacterial infections were low for IGHy and similar to IVIG and SCIG.

- $97.2 \%$ of IGHy infusions in the Phase III study and extension period were completed without slowing, interrupting, or discontinuing administration, and $82.5 \%$ required only a single infusion site.

- Treatment of pediatric patients with IGHy in intervals of 3 or 4 weeks maintained protective trough levels, prevented infections and was well-tolerated.

Anti-PH20 immunogenic response

- IGHy is associated with transient anti-rHuPH20 antibody development in some patients, but the anti-rHuPH20 antibodies are non-neutralizing and without clinical consequences.

Real-world use of IGHy

- IgG replacement therapy is characterized by variation in doses, dosing intervals and lgG trough levels required to protect against infection; individualization of treatment regimens is required.

- IGHy provides an option for individualized treatment that is preferred by many patients over IVIG or conventional SCIG and minimizes the impact of IgG replacement therapy on patients' lives. 


\section{References}

Papers of special note have been highlighted as: $\bullet$ of interest; $\bullet \bullet$ of considerable interest

1 Kobrynski L. Subcutaneous immunoglobulin therapy: a new option for patients with primary immunodeficiency diseases. Biologics 6 , 277-287 (2012).

2 Modell V, Knaus M, Modell F, Roifman C, Orange J, Notarangelo LD. Global overview of primary immunodeficiencies: a report from Jeffrey Modell Centers worldwide focused on diagnosis, treatment and discovery. Immunol. Res. 60(1), 132-144 (2014).

3 Bousfiha A, Jeddane L, Al-Herz W et al. The 2015 IUIS phenotypic classification for primary immunodeficiencies. J. Clin. Immunol. 35(8), 727-738 (2015).

4 Perez EE, Orange JS, Bonilla F et al. Update on the use of immunoglobulin in human disease: a review of evidence. J. Allergy. Clin. Immunol. 139(3S), S1-S46 (2017).

5 Bousfiha AA, Jeddane L, Ailal F et al. Primary immunodeficiency diseases worldwide: more common than generally thought. J. Clin. Immunol. 33(1), 1-7 (2013).

6 Kobrynski L, Powell RW, Bowen S. Prevalence and morbidity of primary immunodeficiency diseases, United States 2001-2007. J. Clin. Immunol. 34(8), 954-961 (2014).

7 Wasserman RL. Progress in gammaglobulin therapy for immunodeficiency: from subcutaneous to intravenous infusions and back again. J. Clin. Immunol. 32(6), 1153-1164 (2012).

- Reviews immunoglobulin G (IgG) replacement therapy, including recombinant human hyaluronidase-facilitated subcutaneously administered IgG (IGHy) in patients with primary immunodeficiencies (PID).

8 Bruton OC. Agammaglobulinemia. Pediatrics 9(6), 722-728 (1952).

9 Long AA, Denburg JA, Dent PB. Hypogammaglobulinemia: therapeutic rationale. CMAJ 137(9), 793-797 (1987).

10 Roifman CM, Levison H, Gelfand EW. High-dose versus low-dose intravenous immunoglobulin in hypogammaglobulinaemia and chronic lung disease. Lancet 1(8541), 1075-1077 (1987).

11 Yong PL, Boyle J, Ballow $\mathrm{M}$ et al. Use of intravenous immunoglobulin and adjunctive therapies in the treatment of primary immunodeficiencies: a working group report of and study by the Primary Immunodeficiency Committee of the American Academy of Allergy, Asthma, and Immunology. Clin. Immunol. 135(2), 255-263 (2010).

12 Orange JS, Grossman WJ, Navickis RJ, Wilkes MM. Impact of trough IgG on pneumonia incidence in primary immunodeficiency: a meta-analysis of clinical studies. Clin. Immunol. 137(1), 21-30 (2010).

13 Shabaninejad H, Asgharzadeh A, Rezaei N, Rezapoor A. A comparative study of intravenous immunoglobulin and subcutaneous immunoglobulin in adult patients with primary immunodeficiency diseases: a systematic review and meta-analysis. Expert Rev. Clin. Immunol. 12(5), 595-602 (2016).

14 Vivaglobin (immune globulin subcutaneous [human]), full prescribing information. CSL Behring GmbH, Marburg, Germany (2006).

15 Espanol T, Prevot J, Drabwell J, Sondhi S, Olding L. Improving current immunoglobulin therapy for patients with primary immunodeficiency: quality of life and views on treatment. Patient Prefer Adherence 8, 621-629 (2014).

-. Describes patient and caregiver preferences for and satisfaction with intravenously administered versus subcutaneously administered IgG replacement therapy for PID.

16 Wasserman RL, Melamed I, Stein MR et al. Recombinant human hyaluronidase-facilitated subcutaneous infusion of human immunoglobulins for primary immunodeficiency. J. Allergy Clin. Immunol. 130(4), 951 e911-957 e911 (2012).

-. Reports final results of the IGHy Phase III trial.

17 Duran-Reynals F. The effect of extracts of certain organs from normal and immunized animals on the infecting power of vaccine virus. $J$. Exp. Med. 50(3), 327-340 (1929).

18 Frost GI. Recombinant human hyaluronidase (rHuPH20): an enabling platform for subcutaneous drug and fluid administration. Expert Opin. Drug Deliv. 4(4), 427-440 (2007).

19 Bookbinder LH, Hofer A, Haller MF et al. A recombinant human enzyme for enhanced interstitial transport of therapeutics. J. Control. Rel. 114(2), 230-241 (2006).

- Describes engineering of recombinant human hyaluronidase $(\mathrm{rHuPH} 20)$ and development for use in combination with IgG for PID.

20 Lathrop WF, Carmichael EP, Myles DG, Primakoff P. cDNA cloning reveals the molecular structure of a sperm surface protein, PH-20, involved in sperm-egg adhesion and the wide distribution of its gene among mammals. J. Cell Biol. 111(6 Pt 2), 2939-2949 (1990).

21 Suez D, Stein M, Gupta S et al. Efficacy, safety and pharmacokinetics of a novel human immune globulin subcutaneous, $20 \%$ in patients with primary immunodeficiency diseases in North America. J. Clin. Immunol. 36(7), 700-712 (2016).

22 Borte M, Krivan G, Derfalvi B et al. Efficacy, safety, tolerability and pharmacokinetics of a novel human immune globulin subcutaneous, 20\%: a Phase II/III study in Europe in patients with primary immunodeficiencies. Clin. Exp. Immunol. 187(1), 146-159 (2017).

23 Thompson CB, Shepard HM, O'Connor PM et al. Enzymatic depletion of tumor hyaluronan induces antitumor responses in preclinical animal models. Mol. Cancer Ther. 9(11), 3052-3064 (2010). 
24 Kang DW, Jadin L, Nekoroski T, Drake FH, Zepeda ML. Recombinant human hyaluronidase PH20 (rHuPH20) facilitates subcutaneous infusions of large volumes of immunoglobulin in a swine model. Drug Deliv. Transl. Res. 2(4), 254-264 (2012).

25 Baker MP, Reynolds HM, Lumicisi B, Bryson CJ. Immunogenicity of protein therapeutics: the key causes, consequences and challenges. Self Nonself 1(4), 314-322 (2010).

26 Rosengren S, Dychter SS, Printz MA et al. Clinical immunogenicity of rHuPH20, a hyaluronidase enabling subcutaneous drug administration. AAPS J. 17(5), 1144-1156 (2015).

-• Demonstrates the immunogenicity of rHuPH20 and the prevalence of anti-PH20 antibodies in the general population.

27 Zhou C, Kang W, Baba T. Functional characterization of double-knockout mouse sperm lacking SPAM1 and ACR or SPAM1 and PRSS21 in fertilization. J. Reprod. Dev. 58(3), 330-337 (2012).

28 Hardy CM, Clydesdale G, Mobbs KJ et al. Assessment of contraceptive vaccines based on recombinant mouse sperm protein PH20. Reproduction 127(3), 325-334 (2004).

29 Pomering M, Jones RC, Holland MK, Blake AE, Beagley KW. Restricted entry of IgG into male and female rabbit reproductive ducts following immunization with recombinant rabbit PH-20. Am. J. Reprod. Immunol. 47(3), 174-182 (2002).

30 Baba D, Kashiwabara S, Honda A et al. Mouse sperm lacking cell surface hyaluronidase PH-20 can pass through the layer of cumulus cells and fertilize the egg. J. Biol. Chem. 277(33), 30310-30314 (2002).

31 Veneziale RW, Printz MA, Jorge M et al. Nonclinical studies to assess possible effects of antibodies to rHuPH20 on the endogenous counterpart. Presented at: 16th Bienniel Meeting of the European Society for Immunodeficiencies.Prague, Czech Republic, 29 October-1 November, 2014.

32 Rosengren S, Huang L, Jadin L et al. Antibodies to recombinant human hyaluronidase (rHuPH20) in PID subjects treated with HyQvia are qualitatively similar to those in a subset of the normal healthy population. Presented at: 16th Biennial Meeting of the European Society for Immunodeficiencies. Prague, Czech Republic, 29 October-1 November 2014 (Poster 199).

33 Wasserman RL. Overview of recombinant human hyaluronidase-facilitated subcutaneous infusion of IgG in primary immunodeficiencies. Immunotherapy 6(5), 553-567 (2014).

34 Shpilberg O, Jackisch C. Subcutaneous administration of rituximab (MabThera) and trastuzumab (Herceptin) using hyaluronidase. Br. J. Cancer 109(6), 1556-1561 (2013).

35 Hamizi S, Freyer G, Bakrin N et al. Subcutaneous trastuzumab: development of a new formulation for treatment of HER2-positive early breast cancer. Onco Targets Ther. 6, 89-94 (2013).

36 Davies A, Merli F, Mihaljevic B et al. Efficacy and safety of subcutaneous rituximab versus intravenous rituximab for first-line treatment of follicular lymphoma (SABRINA): a randomized, open-label, Phase III trial. Lancet Haematol. 4(6), e272-e282 (2017).

37 Misbah S, Sturzenegger MH, Borte M et al. Subcutaneous immunoglobulin: opportunities and outlook. Clin. Exp. Immunol. 158(Suppl. 1), 51-59 (2009).

-. Reports findings from the Phase I/II trial of IGHy replacement therapy in patients with PID.

38 Wasserman RL, Melamed I, Kobrynski L et al. Efficacy, safety and pharmacokinetics of a 10\% liquid immune globulin preparation (GAMMAGARD LIQUID, 10\%) administered subcutaneously in subjects with primary immunodeficiency disease. J. Clin. Immunol. 31(3), 323-331 (2011).

39 Wasserman RL, Melamed I, Stein MR et al. Long-term tolerability, safety and efficacy of recombinant human hyaluronidase-facilitated subcutaneous infusion of human immunoglobulin for primary immunodeficiency. J. Clin. Immunol. 36(6), 571-582 (2016).

-. Reports long-term safety and efficacy data from the IGHy Phase III trial.

40 Bonagura VR, Marchlewski R, Cox A, Rosenthal DW. Biologic IgG level in primary immunodeficiency disease: the IgG level that protects against recurrent infection. J. Allergy Clin. Immunol. 122(1), 210-212 (2008).

41 Lucas M, Lee M, Oksenhendler E, Chapel H. The ratio of mean daily IgG increment/mean daily dose in immunoglobulin replacement therapy in primary antibody deficiencies. J. Allergy Clin. Immunol. Pract. 3(6), 998 e1002-1000 e1002 (2015).

42 Dumas T, Numerof R, Pierre V, Gelmont D, Yel L. Population pharmacokinetic simulations to address time to reach steady state of recombinant human hyaluronidase-facilitated subcutaneous infusion of immunoglobulin (IG) (IGHy) in IG-naïve patients with primary immunodeficiency diseases (PIDD) Presented at: Clinical Immunology Society Annual Meeting. Boston, MA, USA, 14-17 April 2016.

43 US Department of Health and Human Services, US Food and Drug Administration, Center for Biologics Evaluation and Research. Guidance for industry: safety, efficacy and pharmacokinetic studies to support marketing of immune globulin intravenous (human) as replacement therapy for primary humoral immunodeficiency.

www.fda.gov/biologicsbloodvaccines/guidancecomplianceregulatoryinformation/guidances/blood/ucm072130.htm

44 Wasserman RL, Melamed I, Kobrynski L et al. Recombinant human hyaluronidase facilitated subcutaneous immunoglobulin treatment in pediatric patients with primary immunodeficiencies: long-term efficacy, safety and tolerability. Immunotherapy 8(10), 1175-1186 (2016).

-. Reports efficacy and safety of IGHy in pediatric patients from the Phase III trial and extension phase.

45 Yocum RC, Kennard D, Heiner LS. Assessment and implication of the allergic sensitivity to a single dose of recombinant human hyaluronidase injection: a double-blind, placebo-controlled clinical trial. J. Infus. Nurs. 30(5), 293-299 (2007). 
46 Berger M. Incidence of infection is inversely related to steady-state (trough) serum IgG level in studies of subcutaneous IgG in PIDD. J. Clin. Immunol. 31(5), 924-926 (2011).

47 Rosenbach KP, Rabbat CJ, Rozen L, Hughes SM. Real-world use of recombinant human hyaluronidase-facilitated subcutaneous infusion of immunoglobulin (IGHy; HYQVIA) in patients with primary immunodeficiency disorders (PIDD). Presented at: Clinical Immunology Society Boston, MA, USA, 14-17 April 2016.

48 Gruenemeier P, Ernst C, Palagashvili T, Duff K. Retrospective review of needle length and clinical considerations during hyaluronidase-facilitated subcutaneous administration of immunoglobulin (IgHy). Presented at: American College of Allergy, Asthma \& Immunology. San Francisco, CA, USA, 10-14 November 2016 (Poster 259).

49 Gruenemeier P, Ernst C, White C, Duff K. Real-world pediatric experience with hyaluronidase-facilitated subcutaneous immunoglobulin (IGHY) infusion parameters. Ann. Allergy Asthma Immunol. 117(5), S98 (2016).

50 Wasserman RL, Ito D, Xiong Y, Ye X, Bonnet P, Li-McLeod J. Impact of site of care on infection rates among patients with primary immunodeficiency diseases receiving intravenous immunoglobulin therapy. J. Clin. Immunol. 37(2), 180-186 (2017). 